Journal of Applied and Theoretical Physics Research

\title{
Diagnostics and Production of Warm Dense Matter with Multishock Reverberation Compression Technique
}

\author{
Qifeng Chen*, Zhiguo Li, YunjunGu, Jun Zheng, Chengjun Li and Jiangtao Li \\ Laboratory for Shock Wave and Detonation Physics, CAEP, Mianyang, 621900, China
}

\begin{abstract}
*Corresponding author: Qifeng Chen, Laboratory for Shock Wave and Detonation Physics, CAEP, Mianyang, 621900, China; E mail: chenqf_iapcm@aliyun.com

Article Type: Research, Submission Date: 11 October 2017, Accepted Date: 24 October 2017, Published Date: 29 November 2017.

Citation: Qifeng Chen, Zhiguo Li, YunjunGu, Jun Zheng, Chengjun Li and Jiangtao Li (2017) Diagnostics and Production of Warm Dense Matter with Multishock Reverberation Compression Technique. J Apl Theol 1(3): 11-17. doi: https://doi.org/10.24218/jatpr.2017.12.

Copyright: @ 2017 Qifeng Chen, et al. This is an open-access article distributed under the terms of the Creative Commons Attribution License, which permits unrestricted use, distribution, and reproduction in any medium, provided the original author and source are credited.
\end{abstract}

\begin{abstract}
Multishock reverberation compression is an effective means, especially for low Z matter, to create high pressure warm dense states and study material properties under these extreme conditions. To character and interrogate of such state, we have developed a comprehensive diagnostic technique, which was used to determine the multishock compressed states and to obtain the equations of state of warm dense matter (WDM) pressure up to Mbar region. The results are used to validate the existing WDM theoretical model and create new theoretical ones.
\end{abstract}

\section{Introduction}

Warm dense matter (WDM) belongs to a part of high energy density physics, which includes both extensive and rich physics phenomena [1]. Recent technical advances, experimental capabilities and facilities make it possible to create and confine of warm dense states of matter in the laboratory [2] and advanced diagnostics required for the characterization and interrogation of such states [3]. These experimental capabilities include radiation-synchrotron sources [4], energetic materials [5-8], high power lasers [9-16], particle beams [17,18], Z-pinch devices [19-23], and mechanical impact techniques such as utilized in gas-gun launchers [24-28]. WDM is an important state of the evolution and presence of matters in inertial confinement fusion (ICF) and heavy-ion fusion [29]. In particular, thermodynamic, optical, and radiated characteristic of warm dense matter plays an important role in determining for the macro fluid movement of matter and determining for the energy transportation and transfer in the interactions of radiated field with matter in the evolution process. Therefore, further investigation of the properties of warm dense matter and precision improvements on its related parameters, such as equations of state and radiation transportation, are of science significant and applied background in many research fields such as earth's and planetary interior structure $[30,31]$.

The several of experiments have been used as verification and validation of the calculated model of physical properties of warm dense matter. Multiple compressed states achieved in the laboratory can provide a chance of checking the existing WDM theoretical model and creating new theoretical ones. In our experiments, warm dense matter was created by twostage light-gas gun driven, which produce much longer timescales (micro-second) and much larger sizes (grams or $\mathrm{cm}^{3}$ volumes) of WDM with uniform, constant density, pressure, and temperature that can be achieved and sustained, allow a better diagnosis of WDM with these temporal and spatial scales. As well known, multishock measurements are a useful way to assess the calculated models since they amplify the small difference in the principal Hugoniot curve. Use light-gas gun as a drive for WDM, the process of multishock reverberation compressions is implemented by the confinement of two high shock impedances. However, diagnostics of multi-compressed states is a very difficult event. It is needed elaborate to design the special target and the diagnostic system based on interrogation of WDM state. In order to determine experimentally the multiple shock states, a diagnostic approach with the Doppler pins system (DPS), the multi-wavelength channel optical transience radiance pyrometry (MCOP), and streak optical pyrometer (SOP) was used to probe multiple shocks in the WDM, that combines multiple diagnostics enables us to implement (1) an absolute measurement of the single shock state of gas sample for transparent or partiallytransparent of the first-shocked sample for probe lasers, (2) the direct measurements of multishock states, (3) It also provides an experimental paradigm for combining multiple diagnostics that increases the experimental observables. Thus we get improved information and expanded understanding of WDM under 
Citation: Qifeng Chen, Zhiguo Li, YunjunGu, Jun Zheng, Chengjun Li and Jiangtao Li (2017) Diagnostics and Production of Warm Dense Matter with Multishock Reverberation Compression Technique. J Apl Theol 1(3): 11-17. doi: https://doi.org/10.24218/jatpr.2017.12.

strong shock situations. In the following we introduce the design of target, diagnostics, and its application.

\section{The design of target and diagnostics of multi-compressed states}

Strong shocks in the dense gas sample were produced by the impact of a projectile driven by a two-stage light gas gun. WDM was generated by a shock reverberation technique. It is confined a cell with a steel baseplate at impact end and a composite window at other end. For the diagnostics of multishock compress states, the target design is crucially important. It is necessary to avoid the influence of rarefaction and catch-up waves on the compressed samples. In our experiments, the wide-thick ratios of the targets were optimized by using a one-dimensional hydrodynamic code. The exemplary simulated result of the position-time diagram for the trajectories of the shock fronts and interfaces for dense gas $\mathrm{H}_{2}+\mathrm{He}$ mixture multi-shock experiment is shown in Figure 1 , which shows the details of the shock wave reverberations between the steel baseplate and the composite window. In order to determine experimentally the multiple shock states, a diagnostic approach with the DPS, SOP, and MCOP, was used to probe multiple shocks in the WDM. A typical experimental assembly for multiple shock compression of the dense gas in planar geometry of a specific target is shown in the left panel of Figure 2. One end of the cell was sealed with a $\sim 4.0 \mathrm{~mm}$ thick 304 steel baseplate, the opposite end of the cell was sealed a composite window through which the shock emission can be observed. The steel baseplate serves as the shock pusher, which was also used as the pressure standard for impedance matching measurements. The steel sample cell was $\sim 5.0-7.0 \mathrm{~mm}$ thick,

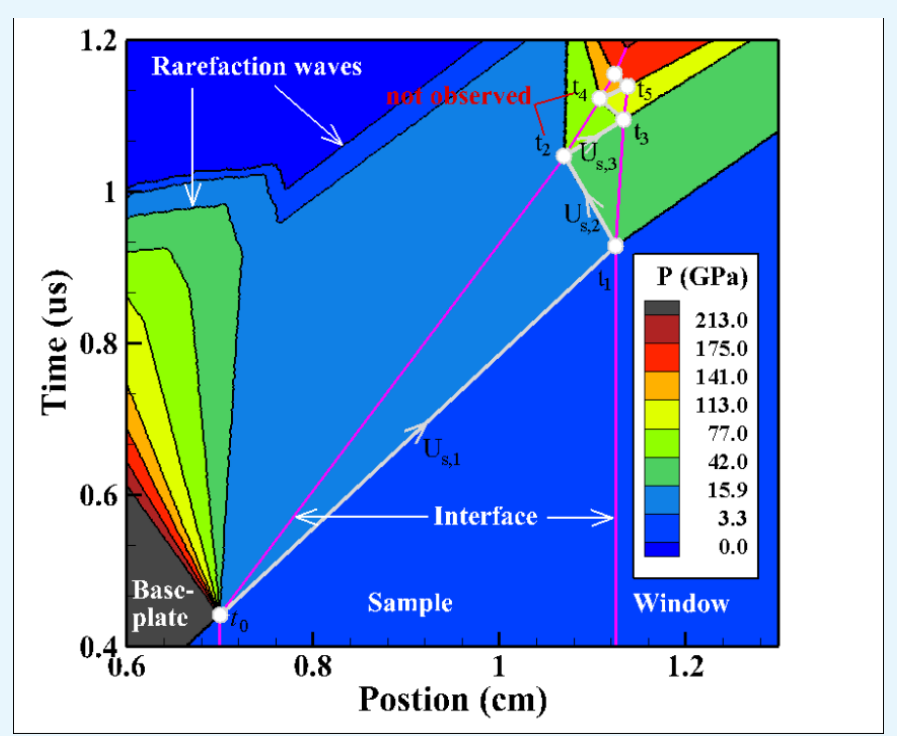

Figure 1: A one-dimensional hydrodynamic simulation position-time diagram indicating trajectories of the shock fronts and interfaces for dense gas multi-shock experiment. $t_{0}, t_{2}, t_{4}, \ldots$ and $t_{1}, t_{3}, t_{5}, \ldots$ are referred to shock arrived at the baseplate/sample and at the sample/film interface, respectively. $U_{s, 1}, U_{s, 2^{2}}, U_{s, 3}, \ldots$ are the shock velocities in the shocked gas sample
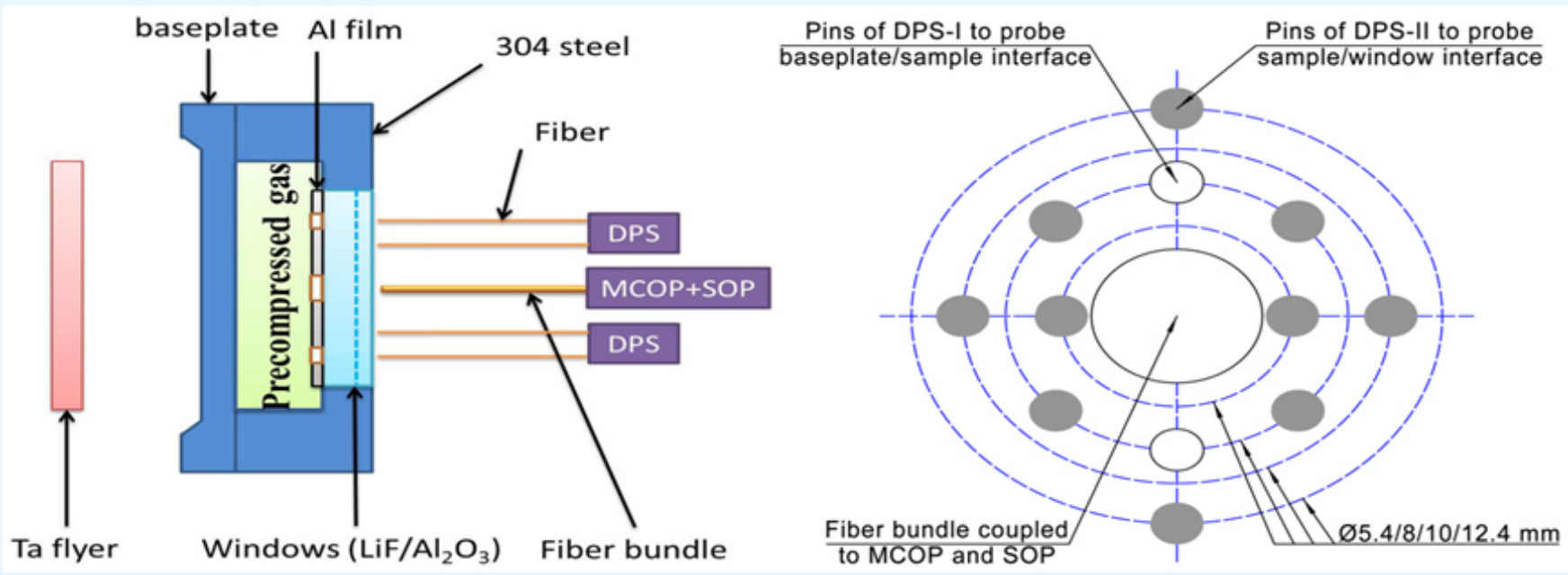

Figure 2: Schematic of the precompressed gas target (left) and arrangement of diagnostic probes (right) used to obtain multishock data for WDM. (Note the drawing is not scale) 
Citation: Qifeng Chen, Zhiguo Li, YunjunGu, Jun Zheng, Chengjun Li and Jiangtao Li (2017) Diagnostics and Production of Warm Dense Matter with Multishock Reverberation Compression Technique. J Apl Theol 1(3): 11-17. doi: https://doi.org/10.24218/jatpr.2017.12.

which was designed according to the different gases. Before shock compression, the gaseous sample was precompressed to a dense state with a pressure of around 20-40 MPa from ambient conditions by pressurization devices to gain high initial density. The initial density $\rho_{0}$ of dense gas sample was measured to an accuracy of $0.2 \%$ by a draining method used in our previous work [32]. The composite window is the most important part of the target, which consists of one layer of $\sim 4.0 \mathrm{~mm}$ thick lithium fluoride (LiF) and one layer of $\sim 2.0 \mathrm{~mm}$ thick sapphire $\left(\mathrm{Al}_{2} \mathrm{O}_{3}\right)$ behind the $\mathrm{LiF}$ to prevent precompression fracturing. The front surface (the side towards the flyer) of LiF has a $\sim 0.12 \mathrm{~mm}$ thick aluminum (Al) film, which serves as a reflective interface for velocity interferometry to measure the velocity of the sample/ window interface. Aluminum was selected because its shock impedance matches approximately that of LiF. Moreover, apertures, one with a diameter of $\sim 4.0 \mathrm{~mm}$ and two with a diameter of $\sim 1.25 \mathrm{~mm}$, were incorporated on the $\mathrm{Al}$ foil and film. The aperture of $4.0 \mathrm{~mm}$ in diameter was designed to allow the light emitted from the shocked gas samples to be measured. And the apertures of $1.25 \mathrm{~mm}$ in diameter were designed to allow the probe lasers of the velocity interferometer devices to pass through for measuring the velocity of the baseplate/sample interface or shock wave front. In our experiments, strong planar shock waves were generated from the impact of high velocity tantalum (Ta) flyers into precompressed gas targets. The waves then reverberated between two higher shock impedances (the steel baseplate and the composite window), to compress gas sample. The Ta flyer, with a thickness of about $\sim 3.0 \mathrm{~mm}$, was accelerated to velocities $4.0-6.5 \mathrm{~km} / \mathrm{s}$ using a two-stage light gas gun with a bore diameter of $30.0 \mathrm{~mm}$. The sizes of the flyer, baseplate, sample cell, and windows were optimized to ensure that the rarefaction and catch-up waves would not compromise the one-dimension character of compression in the optically observed region.

To implement the diagnostics of multiple reverberations compressed state with the MCOP, DPS, and SOP tools, we designed a specific comprehensive diagnostic system, which consists of two sets of velocity interferometer devices-Doppler pins system (DPS), two sets of multi-channel optical pyrometers (MCOPs), and a streak optical pyrometer (SOP) system with a spectrometer coupled to an optical streak camera. The arrangement of these diagnostic devices is shown in the right panel of Figure 2. A 13-fiber bundle positioned at the central area (corresponding to the $\sim 4 \mathrm{~mm}$ diameter aperture) of the rear sapphire window was used to collect the light emitted from the shocked samples and to direct the light to two sets of six-channel pyrometers with different precisions and the SOP system. The six channels of MCOP were centered at six wavelengths between 400 and $850 \mathrm{~nm}$. The relatively large sample in these experiments enabled fibers with diameter of $62.5 \mu \mathrm{m}$ to be used. The numerical aperture (N.A.) of the fibers is 0.275 . Before each shot, the MCOPs and SOP were carefully calibrated using a standard tungsten light source for shock temperature measurements. This calibration is similar to that described in the previous work [26]. The twelve pins connected to the DPS via fibers were distributed symmetrically in four rings of diameters $\sim 5.0, \sim 8.0, \sim 10.0$, and $\sim 12.0 \mathrm{~mm}$, and used to launch the probe laser beams from DPS operating at a wavelength of $1550 \mathrm{~nm}$ and to collect the light returning to the DPS. The probe laser beams from DPS-I were directed onto the baseplate/sample interface or shock wave front through the $1.25 \mathrm{~mm}$ diameter apertures to measure its velocity; the probe laser beams from DPS-II were directly reflected by the Al reflecting film to measure the velocity of sample/LiF interface. The velocities of the baseplate/sample and sample/LiF interfaces were determined, with an accuracy of $0.5 \%$ via the Doppler shift interference fringes of the reflected light. These measurements enable us to obtain all the required variables of shock velocity, particle velocity, pressure, density, and temperature.

\section{Technique's applications}

Dynamic compression of dense gases through shock wave experiments has been paramount in guiding our understanding of the high- $P$ response of WDM, and in the evaluation of emerging computational techniques. The above description target and the comprehensive diagnostic system were applied to interrogate the multi-shock compressed states of WDM, which can be determined from the measured shock radiation histories combining the particle velocity profiles. The multishock temperatures were obtained from the measured radiation histories and the calibrations. The following discussion will be derived into (1) the transparent or partially-transparent of the first-shocked sample for probe lasers under the condition of window maintaining transparent (2) opaque of the first-shocked sample for probe lasers and the window's transparent losing after the first-shock.

\section{The transparent or partially-transparent of the first-shocked sample for probe lasers and the window maintaining transparent}

Firstly, we discuss the situation that the evolution of shock temperature of gas sample is not really high so that the window will maintain transparency in multi-shock process. Figure 3 shows a typical experimental record for this situation, which was obtained from the multi-shock compression experiments for the low- $Z$ gas sample (deuterium-helium mixture) with a pressure of $\sim 20 \mathrm{MPa}$ and an ambient temperature, driven by a $5.13 \mathrm{~km} / \mathrm{s}$ Ta flyer. Figure 3 (a) and (b) are the time-resolved light radiation history of shocked $\mathrm{D}_{2}-\mathrm{He}$ mixtures recorded by two sets of MCOP. MCOP-I provides a clear indication of shock arrival time at baseplate/sample interface $\left(t_{0}\right)$ and sample/LiF interface $\left(t_{1}\right)$, i.e., the first shock. According to the first shock transit time $\Delta t$ $=t_{1}-t_{0}$ and the initial thickness of sample cell, the first shock velocity $U_{s, 1}$ could be determined. After time $t_{1}$, there is still information regarding the multi-shock reverberations, which is beyond the scale of MCOP-I and is measured by MCOP-II as shown in Figure 3(b). Each time the odd shock reached the sample/LiF interface $\left(t_{1}, t_{2}, t_{3} \ldots\right)$, it was reflected by the high 
Citation: Qifeng Chen, Zhiguo Li, YunjunGu, Jun Zheng, Chengjun Li and Jiangtao Li (2017) Diagnostics and Production of Warm Dense Matter with Multishock Reverberation Compression Technique. J Apl Theol 1(3): 11-17. doi: https://doi.org/10.24218/jatpr.2017.12.

impedance LiF and compressed the sample once more, which caused a rise in MCOP-II signals. Therefore, each flat region in MCOP-II after $t_{1}$ consists of two compression processes. For instance, the time interval $t_{2}-t_{1}$ consists of the $2^{\text {nd }}$ and $3^{\text {rd }}$ shock compression processes. In this case, the multi-shock states can't be directly obtained from MCOP signals.

Figure 3(c) shows the velocity histories of baseplate/sample and sample/LiF interfaces obtained from DPS-I and DPS-II, respectively. When the shock broke out into the sample from the baseplate $\left(t_{0}\right)$, the baseplate/sample interface accelerated up to a steady speed, i.e., the particle velocity $U_{p, 1}$ behind the first shock. In the present case that the first-shocked sample is transparent for the $1550 \mathrm{~nm}$ probe lasers, the probe lasers of DPS-I can penetrate through the first shocked sample and reflect from the baseplate/sample interface, so $U_{p, 1}$ can be detected by DPS-I, i.e., the velocity shown in Figure 3(c) corresponding to the first long flat region of the DPS-I signal. The first shock pressure and density can be determined by the Hugoniot state with the

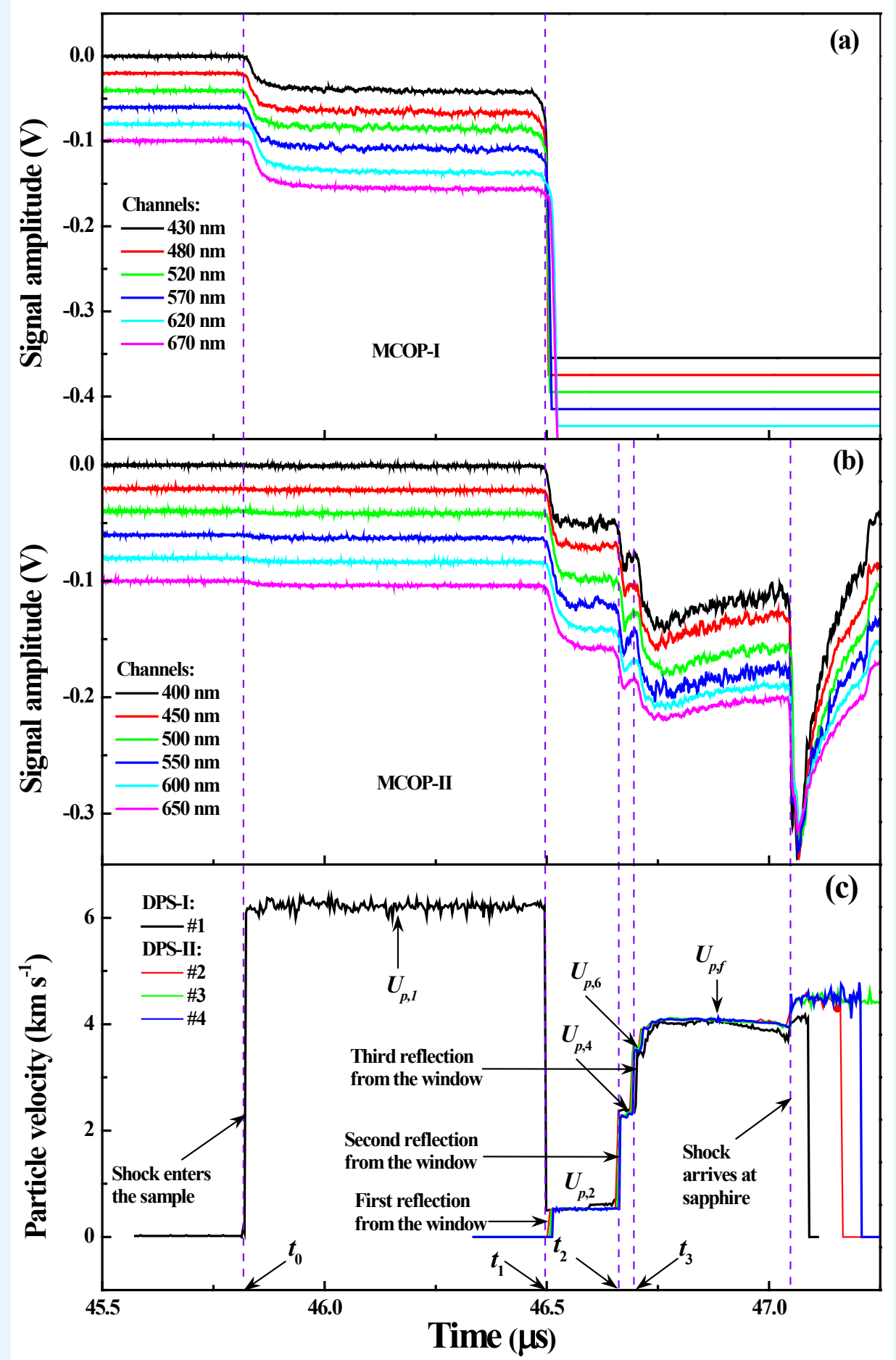

Figure 3: The typical experimental record with MCOP and DPS for the transparent of the first-shocked sample for probe lasers under the condition of window maintaining transparent 
Citation: Qifeng Chen, Zhiguo Li, YunjunGu, Jun Zheng, Chengjun Li and Jiangtao Li (2017) Diagnostics and Production of Warm Dense Matter with Multishock Reverberation Compression Technique. J Apl Theol 1(3): 11-17. doi: https://doi.org/10.24218/jatpr.2017.12.

measured shock velocity $U_{s, 1}$ and particle velocity $U_{p, 1}$.

Secondly, we discuss the case that the first-shocked sample is partially transparent for the probe lasers, part of the DPS-I probe laser penetrates through the first shocked sample and reflect from the baseplate/sample interface, the rest will be directly reflected by the shock front. In this case, DPS-I measures both the apparent particle velocity $U_{p, 1}$ and shock velocity $U_{s, 1}$, which can be corrected according to the refractivity of initial gas sample for $1550 \mathrm{~nm}$ [32]. Figure 4 shows a typical experimental record for this situation, which was obtained from the multi-shock compression experiments for the deuterium-helium mixture with a pressure of $\sim 40 \mathrm{MPa}$ and an ambient temperature, driven by a $5.52 \mathrm{~km} / \mathrm{s}$ Ta flyer.

The signals of the DPS also present a clear physical picture of shock reverberations. Each time the odd shock arrived at the sample/LiF interface, it was reflected by $\mathrm{LiF}$ and compressed the sample once more (the even shock). At the same time, another shock was generated and transmitted through the LiF, while

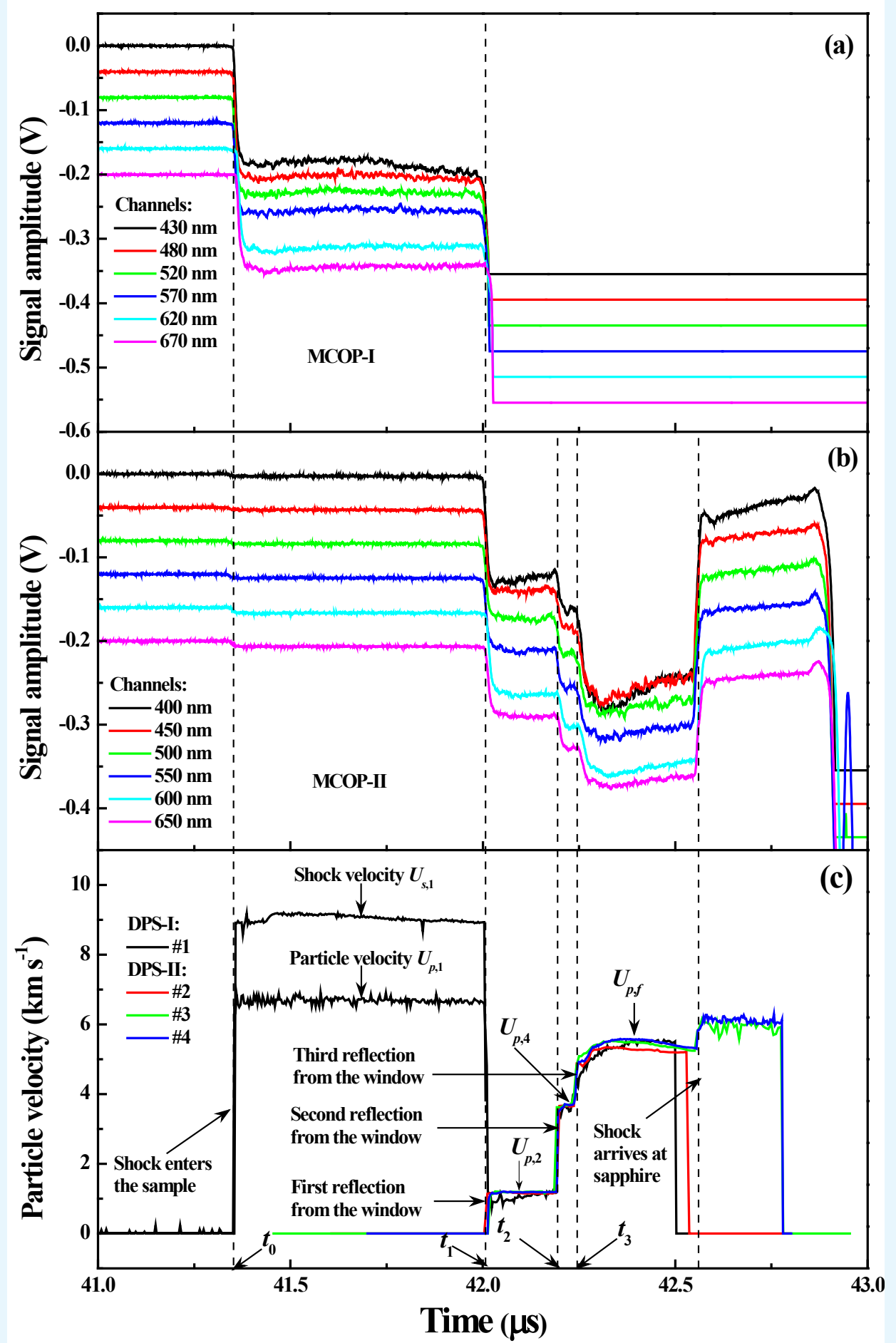

Figure 4: The typical experimental record with MCOP and DPS for the partially-transparent of the first-shocked sample for probe lasers under the condition of window maintaining transparent 
Citation: Qifeng Chen, Zhiguo Li, YunjunGu, Jun Zheng, Chengjun Li and Jiangtao Li (2017) Diagnostics and Production of Warm Dense Matter with Multishock Reverberation Compression Technique. J Apl Theol 1(3): 11-17. doi: https://doi.org/10.24218/jatpr.2017.12.

the motion of the sample/LiF interface, i.e., the particle velocity of the even shock $\left(U_{p, 2}, U_{p, 4}, U_{p, 6,} \ldots\right)$, was instantly detected by DPS-II. The mention particle velocities have been all corrected by the refractivity of the LiF window [33]. It is noticed that the shock reflection times recorded by DPS-II is in reasonable good agreement with that by MCOP-II, which insures the accuracy of the experimental measurements. The multiple compression processes repeated until the sample reached a peak or final state $\left(U_{p, f}\right)$. The multi-shock states including shock velocities, pressures, densities, and compressions, can be directly determined by the impedance matching method with the measured shock reflection times, particle velocities, together with the measured first shock state. The detailed deduction of multi-shock states may see in Ref.[33].

From the signals of MCOP, we can obtain the spectral radiance of the shocked $\mathrm{D}_{2}-\mathrm{He}$ sample by normalizing MCOP signals to the radiation intensities according to the calibrations. The shock temperatures can be obtained by fitting the six-channel data to the Planck gray-body spectrum [26],

$$
I_{\exp }(\varepsilon, \lambda, T)=\varepsilon I_{p l}(\lambda, T)=\frac{\varepsilon C_{1}}{\lambda^{5}} \frac{1}{\exp \left(C_{2} / \lambda T\right)-1},
$$

where $\lambda$ is the wavelength, $\varepsilon$ is the effective emissivity, $T$ is the temperature, and $C_{1}$ and $C_{2}\left(C_{1}=3.7418 \times 10^{-16} \mathrm{~W} \cdot \mathrm{m}^{2} \cdot \mathrm{Sr}^{-1}, C_{2}=\right.$ $\left.1.4388 \times 10^{-2} \mathrm{~m} \cdot \mathrm{K}\right)$ are the first and second radiation coefficients, respectively. The values of $\varepsilon$ and $T$ can be determined by a nonlinear least-squares method. The long radiance "plateau" recorded by MCOP-I in Figure 3(a) can be used for the first shock temperature deduction, whereas the two radiance "plateau" after $t_{1}$ can be used for the $2^{\text {nd }}$ and $4^{\text {th }}$ shock temperature deductions.

\section{The opaque of the first-shocked sample for probe lasers and the window's transparent losing}

If the temperature of first-shocked gaseous sample is relatively higher, the window will lose large partly transparency due to the high temperature ablation. In this case, the spectral radiance history recorded by MCOP will be different from that in section III. A. Figure 5 shows the experimental record of the multishock compression experiments for the gas neon $(\mathrm{Ne})$ with a pressure of $\sim 40 \mathrm{MPa}$ and an ambient temperature, driven by a $4.69 \mathrm{~km} / \mathrm{s}$ Ta flyer. Figure 5(a) is the time-resolved light radiation of shocked Ne recorded by MCOP, which also provides a clear indication for the first shock process $\left(t_{1}-t_{0}\right)$. As the first shock reaches the sample/LiF window at time $t_{1}$, a falling edge indicates that the sample/LiF window interface will be partially opaque due to the thermal ablation of the front surface of LiF window caused by the first-shocked high- $T \mathrm{Ne}$ (the $1^{\text {st }}$ shock temperature is nearly $20 \mathrm{kK}$ ). In this case, the first shock is sufficiently strong, so the compressed sample becomes opaque for the probe lasers of velocity interferometers. The probe lasers of DPS-I will be directly reflected by the shock front, and therefore DPS-I measures the first shock velocity $U_{s, 1}$, i.e., the velocity shown in Figure 5(b) corresponding to the first long flat region of the DPS-I signal. The particle velocity $U_{p, 1}$ needs to be derived by the impedance matching method. Then the first shock pressure and density can be determined by the Hugoniot state with $U_{s, 1}$ and $U_{p, 1}$. The multi-shock processes recorded by DPS-II are similar to that in section III. A, so the multi-shock states can be determined directly from the experimental recordings with the impedance matching approach. The detailed deduction of multi-shock states may find out in Ref. [34].

We can also derive the shock temperatures from MCOP signals with the method described in the situation III. A. However, only the first long radiance "plateau" recorded by MCOP in Figure 5(a) can be used for the first shock temperature deduction due to the $\mathrm{LiF}$ window large partially lost transparency after time $t_{1}$.

We applied the above mention diagnostic technique and method to perform the experiments on warm dense $\mathrm{H}_{2}, \mathrm{D}_{2}, \mathrm{He}$ [33], $\mathrm{H}_{2}+\mathrm{He}$ [26], $\mathrm{D}_{2}+\mathrm{He}$ [32], $\mathrm{Ne}, \operatorname{Ar}[34,35]$, and $\mathrm{Xe}[36,37]$ over several MPa to Mbar pressure region. The two-, four-, six-, and ten-shock in $\mathrm{Xe}, \mathrm{Ar}, \mathrm{Ne}$, and low- $\mathrm{Z}$ gas $\left(\mathrm{H}_{2}, \mathrm{D}_{2}, \mathrm{He}, \mathrm{H}_{2}+\mathrm{He}\right.$, $\mathrm{D}_{2}+\mathrm{He}$ ) reverberate back and forth between the two high shock impedance of base-plate and window was directly observed in our experiments. And that it has been implemented to compress dense gas arriving at a warm dense region from shock-adiabatic to quasi-isentropic compression paths.

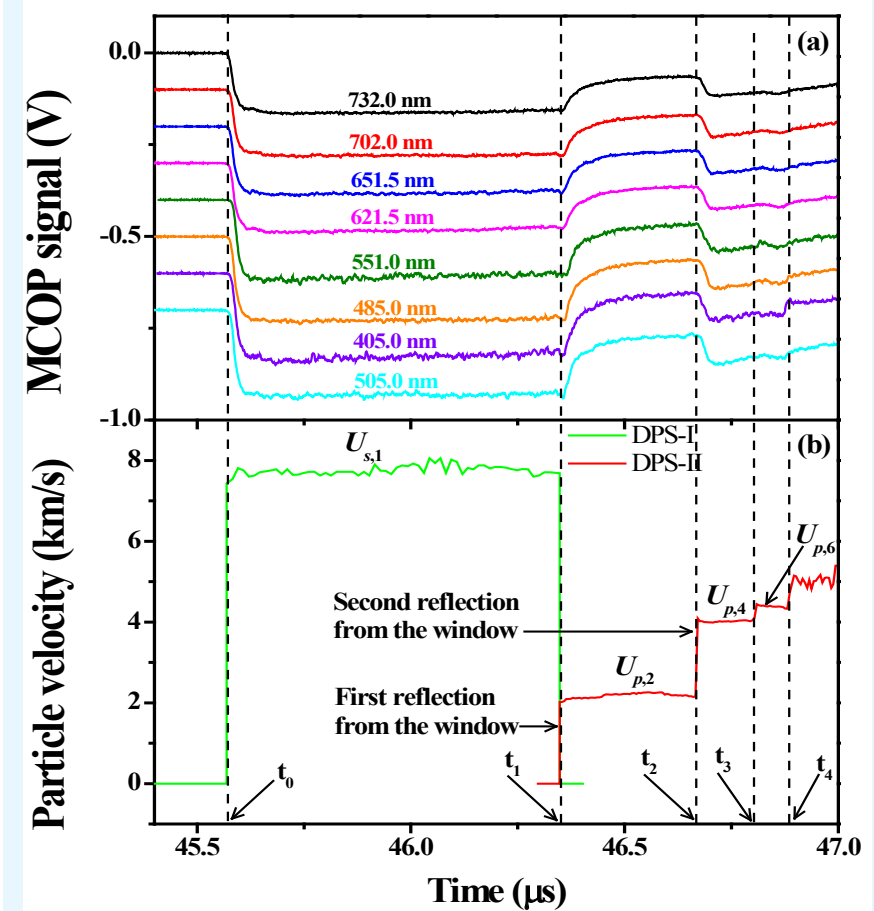

Figure 5: The typical experimental record with MCOP and DPS for the opaque of the first-shocked sample for probe lasers under the window's transparent losing after the first shock

\section{Remark and outlook}

The diagnostics of the multiple shock states in shock reverberation experiments become feasible in virtue of the recent advances in the shock wave measurement techniques. The present measurement and diagnostic technique are of significant for shock wave physics. The obtained shock data are of interest for the 
Citation: Qifeng Chen, Zhiguo Li, YunjunGu, Jun Zheng, Chengjun Li and Jiangtao Li (2017) Diagnostics and Production of Warm Dense Matter with Multishock Reverberation Compression Technique. J Apl Theol 1(3): 11-17. doi: https://doi.org/10.24218/jatpr.2017.12.

development of the thermodynamic properties of the WDM. In particular, using gas gun as a driver for WDM, it would produce uniform large sizes and sustain WDM for long time-scales, allow a better diagnosis of WDM with these temporal and spatial scales. The designed comprehensive diagnostic system was applied to determine experimentally the multiple shock states. It can be implement a target optimized for multi-objective probing in one shot and also provide an experimental paradigm that increases the experimental throughput, decreases cost, and allows for a more complete set of experimental observables. This technique has applied to a wide variety of species and WDM conditions, such as inert gas ( $\mathrm{He}, \mathrm{Ne}, \mathrm{Ar}, \mathrm{Xe}), \mathrm{H}_{2}, \mathrm{D}_{2}, \mathrm{H}_{2}+\mathrm{D}_{2}, \mathrm{He}+\mathrm{H}_{2}, \mathrm{He}+\mathrm{D}_{2}$ mixture gases. The experimental results are used to validate our developed self-consistent fluid variational theoretical model [38], to check the existing WDM theoretical model, and to create new theoretical ones. As an example, if one could reliably measure the thermal and electronic conductivities and reflectivity under these unique conditions, while simultaneously measuring the density, pressure, and temperature, the data set obtained would challenge and expand our current thinking the equation of state of WDM in this regime. Thus, there is a significant need to develop capabilities for creating and diagnosing this state of WDM, both to develop a fundamental understanding of such things as the nature of the matter at the core of planets and earth. But also gain an understanding that can impact applications such as implosion and fusion, and enhanced our understanding of phase transitions, phase diagrams, transport properties, hydrodynamic-radiation transport modeling, and the dynamic response of materials under a wide range of pressure, temperature, strain, and strain rates.

\section{Acknowledgements}

This work was supported by the National Natural Science Foundation of China (Grant Nos. 11674292 and 11504352,), the Science Challenge Project (Grant No. TZ2016001), the Science and Technology Development Foundation of China Academy of Engineering Physics (Grant Nos. 2013A0101001), and the Foundation of Laboratory of Shock Wave and Detonation Physics of China Academy of Engineering Physics (Grant No. 9140C670103150C67289).

\section{References}

1. Kalantar DH, Lee RW, Molitoris JD. UCRL-TR-203844; 2004.

2. Koenig M, Benuzzi-Mounaix A, Ravasio A, Vinci T, Ozaki N, Lepape S, et al. Plasma Phys. Contr. F. 2005; 47:B441.

3. Brambrink E, Amadou N, Benuzzi-Mounaix N, Geissel N, Harmand M, Pelka A, et al. Contrib. Plasm. Phys. 2015; 55:67.

4. Gerward L, Jiang JZ, Staun Olsen J, Recio JM, Waśkowska A. J. Alloy. Compd. 2005; 401:11.

5. Mochalov MA, Il'kaev RI, Fortov VE, Mikhailov AL, Arinin VA, Blikov AO, et al. JETP Lett. 2015; 101:519.

6. Boriskov GV, Bykov AI, II'kaev RI, Selemir VD, Simakov GV, Trunin RF, et al. Phys. Rev. B. 2005; 71:092104.

7. Ternovoi VY, Kvitov SV, Pyalling AA, Filimonov AS, Fortov VE. JETP Lett. 2004; 79:6.
8. Fortov VE, Ternovoi VY, Zhernokletov MV, Mochalov MA, Mikhailov AL, Filimonov AS, et al. JETP. 2003; 97:259.

9. Falk K, Regan SP, Vorberger SP, Crowley BJB, Glenzer SH, Hu SX, et al. Phys. Rev. E. 2013; 87:043112.

10. Regan SP, Falk K, Gregori G, Radha PB, Hu SX, Boehly TR, et al. Phys. Rev. Lett. 2012; 109:265003.

11. Loubeyre P, Brygoo S, Eggert J, Celliers PM, Spaulding DK, Rygg JR, et al. Phys. Rev. B. 2012; 86:144115.

12. Hicks DG, Boehly TR, Celliers PM, Eggert JH, Moon SJ, Meyerhofer DD, et al. Phys. Rev. B. 2009; 79:014112.

13. Eggert J, Brygoo S, Loubeyre P, McWilliams RS, Celliers PM, Hicks DG, et al. Phys. Rev. Lett. 2008; 100:124503.

14. Collins GW, Da Silva LB, Celliers P, Gold DM, Foord ME, Wallace RJ, et al. Science. 1998; 281:1178.

15. Cauble R, Perry TS, Bach DR, Budil KS, Hammel BA, Collins GW, et al. Phys. Rev. Lett. 1998; 80:1248.

16. Da Silva LB, Celliers P, Collins GW, Budil KS, Holmes NC, Barbee Jr TW, et al. Phys. Rev. Lett. 1997; 78:483.

17. Ni PA, Bieniosek FM, Henestroza FM, Lidia SM. Nucl. Instrum. Meth. A. 2014; 733:12.

18. Armstrong MR, Crowhurst JC, Bastea S, Zaug JM, Goncharov AF. Appl. Phys. Lett. 2014; 105:021904.

19. Knudson MD, Desjarlais MP, Becker A, Lemke RW, Cochrane KR, Savage ME, et al. Science. 2015; 46:1455.

20. Bailey JE, Knudson MD, Carlson AL, Dunham GS, Desjarlais MP, Hanson DL, et al. Phys. Rev. B. 2008; 78:144107.

21. Knudson MD, Hanson DL, Bailey JE, Hall CA, Asay JR, Deeney C. Phys. Rev. B. 2004; 69:144209.

22. Knudson MD, Hanson DL, Bailey JE, Hall CA, Asay JR. Phys. Rev. Lett. 2003; 90:035505.

23. Knudson MD, Hanson DL, Bailey JE, Hall CA, Asay JR, Anderson JR. Phys. Rev. Lett. 2001; 87:225501.

24. Gu YJ, Chen QF, Zheng J, Cai LC, Jia OH, Chen ZY, et al. Appl. Phys. 2012; 111:013513.

25. Zheng J, Gu YJ, Chen ZY, Chen QF. Phys. Rev. E. 2010; 82:026401.

26. Gu YJ, Chen QF, Cai LC, Chen ZY, Zheng J, Jing FQ. J. Chem. Phys. 2009; 130:184506.

27. Holmes NC, Ross M, Nellis WJ. Phys. Rev. B. 1995; 52:15835.

28. Nellis WJ, Holmes NC, Mitchell AC, Trainor RJ, Governo GK, Ross M, et al. Phys. Rev. Lett. 1984; 53:1248.

29. Craxton RS, Anderson KS, Boehly TR, Goncharov VN, Harding DR, Knauer JP, et al. Phys. Plasmas. 2015; 22:110501.

30. Nettelmann N, Redmer R, Blaschke D. Phys. Part. Nuclei. 2008; 39:1122.

31. Nettelmann N, Becker A, Holst B, Redmer R. Astrophys. J. 2012; 750:52.

32. Li ZG, Chen QF, Gu YJ, Zheng J, Chen XR. AIP Adv. 2016; 6:105309.

33. Zheng J, Chen QF, Gu YJ, Li JT, Li ZG, Li CJ, et al. Phys. Rev. B. 2017; 95:224104.

34. Chen QF, Zheng J, Gu YJ, Chen YL, Cai LC, Shen ZJ. J. Chem. Phys. 2014; 140:074202.

35. Zheng J, Chen Q, Yunjun G, Li Z, Shen Z. Sci. Rep. 2015; 5:16041.

36. Zheng J, Gu YJ, Chen ZY, Chen QF. Phys. Rev. E. 2010; 82:026401.

37. Zheng J, Chen QF, Gu YJ, Chen ZY. Phys. Rev. E. 2012; 86:066406.

38. Chen Q, Zhang Y, Cai L, Gu Y, Jing F. Phys. Plasmas. 2007; 14:012703. 